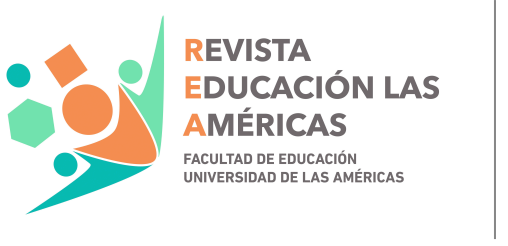

Revista Educación las Américas ISSN: 0719-7128

ccalisto@udla.cl

Universidad de Las Américas

Chile

\title{
Percepciones de los profesionales educativos sobre la Convivencia Escolar
}

\author{
Serey Araneda, Daniel \\ Percepciones de los profesionales educativos sobre la Convivencia Escolar \\ Revista Educación las Américas, vol. 8, 2019 \\ Universidad de Las Américas, Chile \\ DOI: https://doi.org/10.35811/rea.v8i0.4
}

Esta obra está bajo una Licencia Creative Commons Atribución-SinDerivar 4.0 Internacional. 
Artículos

\title{
Percepciones de los profesionales educativos sobre la Convivencia Escolar
}

\author{
Daniel Serey Araneda \\ Universidad de Atacama, Chile, Chile \\ daniel.serey@uda.cl \\ (D) http://orcid.org/0000-0001-6236-836X
}

DOI: https://doi.org/10.35811/rea.v8i0.4

Recepción: 08 Agosto 2019

Aprobación: 23 Septiembre 2019

\begin{abstract}
Resumen:
El presente artículo es parte de los resultados de una investigación (tesis doctoral) sobre la Convivencia Escolar. El objetivo del estudio fue evaluar los discursos que caracterizan y conceptualizan las prácticas educativas de la convivencia escolar, a partir del estudio de las representaciones contenidas en los discursos de los profesionales educativos, mediante un estudio sociocritico, descriptivo y transversal realizado con 272 profesionales educativos de 14 establecimientos municipales en el 2018. La interpretación de los discursos de los participantes develó las distintas "Racionalidades Pedagógicas" que se relacionan con la práctica educativa, experiencias que se dan en la escuela y que están enmarcadas por su modo de ser de la escuela. Para esta investigación se construyeron ocho categorías de análisis. La interpretación de los discursos evidenció las distintas representaciones discursivas que se constituyen en ocho componentes racionales desde sus prácticas educativas en torno a la convivencia, hasta las experiencias que se dan en la escuela y que están enmarcadas por el modo de ser de la escuela.
\end{abstract}

Palabras Clave: cultura, Convivencia Escolar, prácticas educativas, practicas pedagógicas, componentes racionales.

\begin{abstract}
:
This article is part of the results of a research involved in a doctoral thesis based on School Coexistence. The objective of the study was to evaluate the discourses that characterize and conceptualize the educational practices of school life, based on the analysis of the representations contained in the discourses of education professionals, through a socio-critical, descriptive and cross-sectional study. The sample consisted of 272 education professionals from 14 municipal establishments in 2018. The interpretation of the speeches of the participants showed the different "Pedagogical Rationalities" that have connection with the educational practice (experiences that occur in the school and that are framed by the school's behavior). For this investigation, eight categories of analysis were constructed. The interpretation of the speeches evidenced the different discursive representations that constitute eight rational components from their educational practices around coexistence, to the experiences that occur in the school and that are framed by the school's behavior.
\end{abstract}

KEYWORDS: culture, school coexistence, educational practices, pedagogical practices, rational components.

\section{INTRODUCCIÓN}

En Chile el término "Convivencia Escolar" se utiliza para referirse a lo que la literatura anglosajona define como Edad de Oro "durante la Edad Media en que musulmanes, judíos y cristianos fueron capaces de establecer relaciones pacíficas, a pesar de diferencias y tensiones, durante los siete siglos en que los musulmanes gobernaron el sur de España a principios de 1900" (Carbajal Padilla, 2013, p.14). Sin embargo, la convivencia escolar hoy se define "como un espacio de co-construcción en las relaciones interpersonales" (Muñoz, Lucero, Cornejo, Muñoz \& Araya, 2014, p17). Para Carbajal Padilla (2013) "un campo emergente y poco teorizado del cual existe escasa evidencia empírica sobre su implementación en aulas y escuelas. La intención del presente artículo es hacer una contribución a esta discusión” (p.13). La convivencia escolar es un concepto que se constituye en base a un repertorio de ideas, prejuicios y creencias instaladas en la misma escuela, a través de las practicas escolares que construyen y reconstruyen la comunidad escolar en sus interacciones emocionales (Fierro-Evans \& Carbajal-Padilla, 2019). Se entiende que la escuela es un espacio de aprendizaje 
emocional inteligente, potente y esencial, para adquirir desde hábitos a valores que proporcionan el sustento a la formación de ciudadanos democráticos (Serey, 2006).

En este sentido, parece oportuno adentrarse en conocer, en primer lugar, si existe una conceptualización de la Convivencia Escolar propia en las escuelas municipales, a partir de las elaboraciones propias desde sus prácticas pedagógicas. Por una parte, podríamos suponer que, tales prácticas escolares instalarían ciertas conceptualizaciones y significados que constituirían entre todas, un discurso paradigmático, organizado desde los sustratos cognitivos, afectivos y conductuales de cada individuo, como componentes de una cultura en particular que darían sustento a los procesos de la Convivencia Escolar. Por otra parte, si concebimos que la convivencia tiene en sí misma un valor cultural, no sólo como instrumento de resolución de conflictos, sino como re-significante de los recursos didácticos y/o educativos, que posibilita o no a la comunidad escolar la interiorización de valores y hábitos democráticos en su interior y en el mejoramiento de la vida en comunidad (Serey, 2006). A esto, se puede añadir que la convivencia es una interacción de relaciones compleja entre los sujetos que participan de la escuela, soporte de la dinámica que influye en el clima emocional, propio para cada lugar (Cerda, Salazar, Guzmán \& Narváez, 2018), "lo cual llevará a la paradoja de que el estudio de la convivencia esté inundado de investigaciones sobre la violencia, limitando el desarrollo de conocimiento sobre cómo construir la convivencia (Fierro-Evans \& Carbajal-Padilla, 2019, p.11).

Si compartimos la idea de que las instituciones educativas son conceptualizadas teóricamente como un espacio de compromiso y labor formadora a través de la enseñanza, el aprendizaje y la evaluación, tendríamos también que afirmar que su objetivo es sembrar los modelos culturales actuales y por tanto las formas de convivir en espacios no violentos, aunque no carentes de conflictos, que apunten a desarrollar sujetos solidarios, responsables, democráticos y autónomos (Serey, 2019), puesto que la escuela vista como espacio cultural, tiene como objetivo transversal integrar las prácticas de convivencia en la comunidad educativa. Sin embargo, esto no se refleja espontáneamente, sino por el contrario, las conductas agresivas, discriminatorias y el poco respeto por el otro, componen las actitudes más comunes con que la comunidad escolar asume sus conflictos y problemáticas más usuales (MINEDUC, 2010). Incluso hoy, a pesar de haber una Política de Convivencia Escolar, resulta paradójica la falta de un lenguaje concreto, lo que permite que exista confusión y ambigüedad (Fierro-Evans \& Carbajal-Padilla, 2019).

En este sentido, cuando se examina la importancia de mejorar la convivencia, se podría hipotetizar varias intenciones al respecto, entre ellas, la incitación a intervenir para transformar la convivencia, a la mejora como objeto de preocupación, la mejora de la convivencia como proceso que implica la existencia de un dispositivo y un enfoque permanente ..."como si estos esfuerzos supusieran necesariamente una reducción del problema e integrara valores sociales de respeto y buena convivencia" (Ministerio de Educación, 2015, p.5). Lo que corresponde es "dar un sentido pedagógico y transformador a las practicas pedagógicas en torno a la Convivencia Escolar, dejando en paréntesis el cumplimiento formal de los instrumentos relacionados con ella, resaltando el sentido formativo de la escuela/liceo" (Ministerio de Educación, 2015), ya que los propósitos básicos de las escuelas no se constituyen como acciones de socorro de la comunidad escolar, sino que se trata de darle el sentido dialógico comunicativo que proporcione espacio a las interpretaciones propias que cada docente hace de su práctica pedagógica y, en especial, si se trata de la convivencia (Valenzuela, Ahumada, Rubilar, López \& Urbina, 2018).

La escuela y puntualmente, la convivencia dentro de esta, como instrumento esencial para la socialización humana, se ha centrado habitualmente en una visión que potencia los esfuerzos de los profesionales escolares para disciplinar a sus estudiantes bajo el mando de los absolutos racionales (normativa), que no es alcanzado por las propuestas públicas (Serey, 2013). Esto no va con el espíritu de la propuesta publica en Chile de formar seres humanos integrales, respetuosos y valóricos (Ministerio de Educación, 2010), sino que crea divisiones en las interacciones de los distintos estamentos, ya que se influyen y se modifican permanentemente (Ochoa \& Diez-Martín, 2013). 
Nuestro supuesto inicial es que existe una cultura de la convivencia práctica que se da entre toda la comunidad escolar y que es una de las múltiples funciones de la escuela, pues, la convivencia se constituye mediante la creación de ella misma. Esta perspectiva es distinta con tradiciones educativas que se construyen desde la comunidad y que idean la educación como un proceso encaminado a contener las emociones, de modo que no perturben la adquisición de los "buenos modales" lo que haría del niño/a o joven un adulto" bien educado" (Serey, 2006). Estas tradiciones permanecen hasta hoy día y los énfasis siguen estando en la adquisición y representación de ellas, los contenidos y la acumulación de conocimientos por sobre el desarrollo de competencias interpersonales.

En este sentido, el hecho de no visualizar la conceptualización que se tiene sobre la Convivencia Escolar repercute claramente en esta y genera un contexto con tensiones, incomodidad que, en definitiva, interfiere en el trabajo educativo (Güell i Barceló \& Muñoz Redon, 2000). En este sentido, la escuela constituye un espacio predilecto de coexistencia interpersonal, social y organizacional, que sirve de modelo y da sentido a los estilos de relación que se configuran en su interior.

\section{Metodología}

El análisis de la Convivencia Escolar considera como contexto la perspectiva mediadora destinada a establecer la existencia de una cultura de Convivencia Escolar en los sentidos racionales que tienen los profesionales de la educación sobre la misma en Chile. Esta investigación es un estudio empírico teórico de tipo exploratorio descriptivo. El presente estudio tiene un diseño no experimental y de corte transaccional, investigación de tipo Comprensivo-interpretativo.

Tipo y diseño: Se realizaron entrevistas en profundidad de los profesionales educativos para conocer los componentes que constituyen y caracterizan (la conceptualización de) la cultura de la convivencia escolar. Se procedió a realizar un análisis de las respuestas de las entrevistas realizadas a los 271 sujetos, para ello, se utilizó el análisis del discurso, específicamente de contenidos como la técnica de investigación, a partir de los datos de inferencias, reproducibles y válidos, que se aplicaron en el contexto de esta investigación.

Muestra: En esta investigación se seleccionó al 15\% del personal escolar de la Corporación Municipal de Puente Alto que corresponde a 271 de profesionales escolares a través de un muestreo intencional, no probabilístico (Hernández, Fernández y Baptista, 2006). Esta investigación contó con un comité ético, compuesto por cuatro profesionales con grado de Doctor.

Instrumentos: Las técnicas que se emplearon fueron entrevistas semiestructuradas y entrevistas abiertas que se aplicaron a 271 de profesionales escolares. En cuanto al proceso de validación de las entrevistas en profundidad, se elaboró a partir de la revisión teórica y se siguió las etapas comunes (Hernández, Fernández y Baptista, 2006).

- Delimitación de las variables de la investigación.

- Establecimiento de los principales criterios.

- Confección de una entrevista inicial para luego ser validado.

- Confección del cuestionario definitivo tomando en cuenta el proceso de validación mencionado anteriormente.

Procedimiento: El diseño de la investigación implicó las siguientes fases:

a) Fase inicial de investigación: trabajo anticipatorio o de familiarización de objeto y campo. Entrevista indagatoria inicial a encargado de convivencia escolar de la Corporación Municipal de Educacion;

b) Fase investigativa de proceso: aplicación de cuestionario y realización de entrevistas en profundidad y

c) Fase final o de cierre de la investigación: es la fase del trabajo sobre los corpus de información de organización, análisis e interpretación de la información recolectada. La investigación anticipatoria (piloto) se inició en noviembre del año 2018 con la realización de entrevistas a directivos de los 14 establecimientos seleccionados a fin de obtener la autorización para poder llevar a cabo entrevistas en profundidad en los 
meses de noviembre y enero con los 10 sujetos seleccionados. Luego, se entrevistaron 271 sujetos de los 14 establecimientos escolares, durante los meses de abril a agosto en sus propios establecimientos, donde fue posible generar un ambiente adecuado de privacidad y resguardo de los sujetos. El tiempo aproximado de aplicación del instrumento fue de 45 minutos. Los sujetos firmaron un consentimiento informado para participar en la investigación.

Análisis de datos: Esta etapa permitió el análisis de las entrevistas en profundidad y la reconstrucción del discurso colectivo a través de las evidencias reunidas en las grabaciones y posteriores transcripciones para su análisis y discusión de los resultados a partir de la recolección de los datos obtenido en el procedimiento interpretativo o análisis cualitativo.

Las entrevistas en profundidad sobre la temática de Convivencia Escolar permitieron conocer los componentes que constituyen y caracterizan (la conceptualización de) las prácticas educativas de la convivencia, a través de la triangulación y análisis del discurso de los sujetos investigados. En general, nuestro itinerario de investigación permitió producir y validar secuencialmente los componentes conceptuales que se consideran como resultado de esta investigación.

Los resultados muestran un total de ocho categorías que evidencian cómo los discursos docentes operan como dispositivos interaccionales entre la práctica pedagógica y los discursos educativos, lo que permite la instalación y construcción de significados, que contribuyen a instalar la conceptualización de la convivencia escolares, es decir, lo relacional, lo normativo y lo regulativo se instalan desde las mismas instituciones educativas. Estos discursos internos, como dispositivos relacionales, normativos y regulatorios que tienen que ver con la práctica educativa, las relaciones educativas y las interacciones pedagógicas, están mediados desde ciertas lógicas que estarían constituyendo en las instituciones escolares una cultura de Convivencia Escolar.

\section{RESUltados Y DisCUSIÓN}

El estudio de las Racionalidades Pedagógicas propuestas permitió identificar una serie de discursos, que ayudaron a establecer que existe una cultura de la convivencia que interviene en los procesos más relevantes que realizan los profesionales educativos, cuyos contenidos, han posibilitado el establecimiento de ocho dimensiones.

\subsection{Reglamento de Convivencia Escolar (como prescripción permanente)}

Se edifica dentro del estamento adulto, dadas las pautas incriminadas por el contexto en el cual se sitúan la conceptualización del reglamento y de la convivencia como sinónimos. Se observa que algunas de estas reglas son explícitas y otras son tácitas, y existe mayoritariamente en sus discursos, el deseo de construir un espacio escolar de normas y su correcta aplicación, lo que se vive como una necesidad imperiosa, que deja de lado los vínculos entre ellos y la norma legal.

En este escenario, la "vigilancia" al comportamiento se presenta como un mecanismo que previene la transgresión a los límites establecidos en los reglamentos, pero que son percibidos de su falta de cumplimiento al pie de la letra. De la misma forma, la "sanción" cumple una función ilustrativa de lo que no se debe hacer en la escuela para reforzar la adhesión a cierto pacto social implícito de las normas del buen vivir. Si bien, hay un cierto reconocimiento hacia la capacidad que tienen las autoridades para resolver sus conflictos, no se visualiza ni se valora una intervención que favorezca determinado tipo de conflictos. Se considera que se debe exigir la sanción como herramienta pedagógica para mejorar la disciplina (Serey, 2011).

La baja disposición de los apoderados es atribuida como uno de los elementos que influye en la naturaleza de la mala calidad educativa y que gatilla los conflictos que se expresan en distintos ámbitos escolares. Se señala la existencia de una época anterior caracterizada por un ambiente relacional de cercanía, familiaridad, 
compromiso y participación, imagen que contrasta fuertemente con el escenario relacional actual donde se identifica un alto nivel de individualismo y división generándose un clima de tensión y conflictividad (Serey, 2006).

\subsection{Normativas fácticas de la convivencia escolar}

Constituyen un elemento importante en las relaciones escolares para normalizar el conflicto como parte inherente en su discurso, sin que exista un intento por tolerar el conflicto y verlo como una oportunidad desde su gestión.

Los profesionales de la educación no son capaces de ver la relación entre conflicto y convivencia ni entender la convivencia como un proceso, creativo y respetuoso con las partes, de resolver los conflictos, ya sea previendo su producción, ya sea evitando su escalada

El diálogo es irrelevante desde ellos, no existe aprendizaje de los conflictos, solicitan que sus autoridades sean exigentes, sin embargo, según Serey (2013), un modelo basado en la contención emocional no es la solución, por el contrario, ante la ocurrencia de cualquier problema disciplinario, se suelen justificar las actuaciones de los estudiantes de acuerdo a sus condicionantes familiares, sociales, emocionales o económicas por parte de los profesionales del PIE (Programa de Integración Escolar), por lo que se hace muy complejo conseguir un cambio de actitud o de utilizar alternativas que puedan mejorar las responsabilidades, empatía, tolerancia, pues, existiría un rol totalmente paterno y rotulador de la justificación, en este contexto.

Este proceso de transformación de los modelos de intervención demanda una mayor autonomía y reflexión por parte de los docentes respecto de su práctica pedagógica para tener el control de su impacto en la convivencia y para no sólo recibir las directrices de lo que debe ejecutar en la comunidad educativa, pues se exige, que cada sujeto sea consciente y capaz de gestionar elementos importantes de su propia personalidad (empatía cognitiva y emocional, técnicas de afrontamiento), así como elementos interpersonales (comunicación efectiva, negociación manejo de los conflictos, planificar y ejecutar las intervenciones). Al hablar de convivencia hacemos referencia a relacionarnos con los demás, a vivir juntos, como dice Marías (1996), la vida personal es necesariamente interpersonal, es decir, la convivencia hace referencia a la cohesión de grupos y de instituciones: los sistemas de relaciones entre las personas son el núcleo de la convivencia (Ortega, 2007).

\subsection{Gestión institucional de lo normativo}

Lo normativo es la vía por la cual los sujetos de investigación confrontan el clima de las relaciones escolares y buscan apaliar la conflictividad y la violencia escolar, sin embargo, no la visualizan como una perspectiva integral. Es decir, la convivencia no es solo un componente más que apunta a la mejora del clima escolar, sino un conjunto de interacciones y transacciones que se generan en la tarea educativa en una situación espaciotemporal dada.

Para los entrevistados, la convivencia escolar se cristaliza en un factor de equilibrio normativo de las relaciones personales en la escuela, olvidando que a convivir se aprende, y se aprende en cada espacio en que se comparte la vida con otros (Ministerio de Educación, 2005), es decir, en la práctica misma (Maldonado, 2004), sin embargo, como practica activa de la escuela se ha rezagado a un segundo plano, en el que priman los enfoques disciplinarios y la adquisición de normas. En este sentido, la cultura de la convivencia escolar está configurada por elementos formales que dan forma y calidad a la convivencia y que inciden en el nivel de pertenencia de los miembros de la comunidad educativa, es decir, la convivencia en este caso no se basa en un equilibrio en el que los miembros de un grupo comparten metas y normas, como lo plantea Acosta (2003). Si bien, estamos de acuerdo con que deben existir nomas mínimas, cuando un colectivo concluye 
cuales son estas normas, es necesario que sean reconocidas y validadas por todos y todas, incluso por aquellos apoderados definidos como poco comprometidos, que se garantice su reconocimiento y por lo mismo, que el colectivo sea parte de su gestión (elaboración, aplicación, seguimiento, revisión).

\subsection{Relaciones interpersonales e interprofesionales de la convivencia escolar}

La convivencia no es conceptualizada como la práctica de las relaciones entre personas y estas en su entorno, basadas en las actitudes y los valores que ellos mismos ponen en sus interacciones grupales entre ellos con ellos y con sus estudiantes como el respeto, la participación, la práctica de los derechos humanos, la dignidad, la tolerancia, entre otros tantos factores, como un hecho colectivo, que estructure sus actitudes y valores.

Las relaciones son vividas desde una mayor complejidad y heterogeneidad social, consecuencia de la globalización de la que pueden manejar, que llevan a establecer juicios de valor a las personas. Por lo tanto, el discurso sobre el reglamento de la convivencia escolar, como objeto de estudio, es un fenómeno que se complejiza a tal punto que no es puesto en los múltiples factores, agentes y situaciones implicadas en ella (Jimerson \& Furlong, 2006; LLECE, 2002). El colectivo no es capaz de imaginar el reglamento como parte del desarrollo curricular de sus estudiantes ni de la estructura organizativa previamente establecida desde la escuela (Ramírez \& Justicia, 2006).

Se observa mayoritariamente en los discursos de los participantes el deseo de construir un espacio escolar de normas y su correcta aplicación, lo que se considera una necesidad imperiosa, que deja de lado los vínculos entre ellos y la norma legal. Se deja entrever también cómo conviven reglas explícitas con otras que han obtenido su lugar en el uso cotidiano, y como ambos tipos de reglas tienen sus sanciones en los estudiantes y apoderados. Además, existe, como trasfondo, la sensación de la ausencia del reglamento de convivencia escolar, el modo en que es entendido el término "reglamento de convivencia escolar" por los actores entrevistados evidencia el intento por recoger elementos erróneos lo que se traduce en una discrepancia de su conceptualización, esto se debe principalmente porque ya la concepción del término "convivencia escolar" es difusa y no está a la mano de los entrevistados, ya que siempre es necesario hurgar en la conversación para llenar el término con algún sentido, aun cuando, contantemente se señala familiaridad con el término de los entrevistados, pero ellos utilizan el término más como un criterio de valoración que como una distinción que sirva para actuar proactivamente.

Por su parte, existe una concepción de la convivencia escolar predominantemente basada en los problemas con los cuales se la relaciona, pareciera que esta distinción es útil para mirar solo la problemática de la escuela, sin embargo, muchas de las distinciones que usan son las mismas que engloban la "convivencia escolar". Esto se puede interpretar, comprendiendo que el término en sí es usado dentro de la escuela por los miembros, principalmente para englobar significaciones que lo preceden, en lugar de estar aportando distinciones nuevas.

\subsection{Problemas de la Convivencia Escolar}

Desde la perspectiva de los sujetos investigados, el conflicto y los problemas son percibidos como algo de lo que se debe huir. Han conceptualizado que los conflictos surgen entre alumnos de cierto nivel sociocultural y que se les denomina alumnos vulnerables, naturalizando esto y encadenándolo a un solo perfil de estudiantes. Este razonamiento impacta sobre la calidad de la convivencia y en las construcciones simbólicas que circulan en la escuela como creencias absolutas. En esta línea, podríamos decir que el ejercicio y respeto de ciertos derechos y deberes de estos estudiantes constituiría los bordes de lo que se podría calificar como la conceptualización problemática de la convivencia. 
Al conflicto también se lo define como un estado emotivo doloroso, generado por una tensión entre deseos opuestos y contradictorios que ocasiona contrariedades interpersonales y sociales, y en donde se presenta una resistencia y una interacción reflejada muchas veces en el estrés, una forma muy común de experimentarlo (Fuquen Alvarado, 2003).

Esta conceptualización instrumental aplasta la pretensión ilustrada y racionalista de encontrar los mecanismos innovadores para su intervención desde sus raíces, pues, no los deja libre de los condicionamientos lingüísticos de lo que ellos llaman vulnerabilidad escolar y de la falta de interés de cambiar estas conceptualizaciones.

\subsection{Principios y propuestas educativas nacionales para la convivencia}

Se trata del espacio donde se espera que se desplieguen determinados tipos de vínculos, caracterizados por diversos elementos que configuran una red de significación anudada en torno a lo afectivo desde el mismo reglamento de convivencia, sin embargo, no existe conciencia de intervención educativa que permitan mirarse y abordar los conflictos como una problemática que genera las mismas interacciónales entre profesores y alumnos que están lingüísticamente construidas.

Cuando se establece el uso de los manuales de convivencia, se evidencia que los sujetos de estudio esperan sólo recibir las directrices de lo que debe ejecutar para mejorar la convivencia, sin visualizar propuestas interesantes e integradoras por ellos, como si solo fuera responsabilidad de los directivos... "esto permite que se cree una cultura escolar determinada, fortalecida a través de prácticas propias de cada escuela, colegio o liceo, contenidas en el Proyecto Educativo Institucional” (Sandoval, 2014, p.144)

Observamos que los entrevistados no afrontan la convivencia escolar como un proceso comunicatorio de la comunidad educativa, con miras a construir espacios de confianza y de consenso, pues ellos no visualizan la convivencia escolar como el proceso mediante el cual todos los miembros de la comunidad educativa aprenden a vivir con los demás (Vilar Rubiano y Carretero, 2008), pues la convivencia no es sólo la ausencia de conflicto en la escuela, sino que se constituye por las formas de interacción entre los diferentes elementos del sistema educativo y de los diversos aspectos como los procesos y estilos comunicativos prevalentes en la escuela (tanto al interior de ella, como en su relación con la comunidad circundante), que favorece de este modo, la mejora de los aspectos contextuales y organizativos en los que se ejecutan los procesos de enseñanza y aprendizaje. Sin duda que la mejora de la convivencia es uno de los factores de protección más valiosos para prevenir, detener y reducir la violencia (Tuvilla, 2004).

\subsection{Construcciones tipológicas en relación a la convivencia escolar}

Existe un acuerdo generalizado para nuestros entrevistados de que, por un lado, el éxito de los sistemas educativos y de los logros de aprendizaje de los estudiantes dependen de la calidad de la Convivencia Escolar (CE), sin embargo, por otro lado, la atención a la gestión de esta es relativamente pobre, no redunda en la calidad de la educación y se justifica por falta de preparación. En la medida en que los entrevistados se sienten poco preparados para enfrentarla, no se producirá en ellos un cambio cultural como tal, lo que provoca una identidad tanto personal como profesional de ser víctima de los problemas. Las normas de la convivencia escolar

configuran el contexto sobre el cual el profesor chileno debe desenvolverse profesionalmente. Sin embargo, existen otros elementos, puestos en juego por el profesor, que configuran otro plano de acción. Con respecto a ellos, nos referiremos a tres que se encuentran directamente relacionados con la técnica de análisis de incidentes críticos y los objetivos de nuestra investigación: estilo, reflexión y discurso del docente (Nail Kröyer, Muñoz Reyes \& Gajardo Aguayo, 2013). 
Existe una aceptación de la necesidad de elaborar proyectos curriculares que mejoren la convivencia, pero al momento de realizar propuestas, se vuelve a los modelos punitivos, que consideran esencialmente el vigilar y castigar todo incidente disciplinario que quebrante las normativas y que perturbe la normal relación sumisa de los estudiantes de la institución. Ellos esperan de parte de sus autoridades que cuando existe un ambiente disruptivo debe haber inminentemente una sanción disciplinaria.

\subsection{La comunidad educativa como entorno}

La comunidad educativa se conceptualiza, por una parte, desde un rol con carácter profesional en la medida en que su ejercicio se asoció a la posesión de una base de conocimientos relativamente definida, producto de que pasaron por un proceso universitario, sin embargo, por otra parte, necesariamente comparaban su estatus con otras profesiones en donde se tiene menor desgaste y mayor valoración económica y social. Por lo tanto, su identidad brota del modo en cómo internalizan esta visión, y fundan y rehacen significados en torno a ella a lo largo de su vida profesional.

La convivencia escolar no se puede plantear solo como la reducción de los enfrentamientos, el límite de las acciones de maltrato, sino como parte de la proyección satisfactoria para la consecución de los objetivos educativos, en especial para aquellos alumnos de mayor vulnerabilidad social. La gestión institucional de lo normativo no es más que los acuerdos, los mecanismos de control y el compromiso de toda la comunidad con todos aquellos estudiantes con menor arraigo social, es decir, "por lo tanto es necesario y urgente empezar actuar, a definir algunas acciones a realizar, no podemos ser solo espectadores, debemos de ser actores en esta vida, es vital hacer algo que favorezca la convivencia escolar" (Gutiérrez-Méndez \& Pérez-Archundia, 2015, p. 66)

La aplicación de la ley de inclusión por parte de los profesionales de la educación se presenta con algunas reacciones de impotencia en la medida en que son vistas como punitivas y conllevan la eliminación de sus recursos pedagógicos, tales como la expulsión y cancelación de matrícula, hasta entonces sus herramientas profesionales. No obstante, más allá de esto, también es permisible señalar que a los entrevistados les preocupa la pérdida de certezas respecto a los objetivos de su tarea (Van den Berg, 2001).

Se observa, a primera vista en nuestros entrevistados, un mundo escolar que cada día se hace menos amigable para quienes participan en él. A menudo la culpa de esto se atribuye a los estudiantes y sus apoderados, principalmente aquellos catalogados como vulnerables, la que parece una distinción útil para mirar el mundo social de la escuela y su problemática. La convivencia no se exterioriza como un espacio de encuentro y cercanía donde se espera que las partes involucradas asuman ciertas actitudes de colaboración y reciprocidad. Existen ciertas expectativas en relación que un número menor de alumnos podría ser la solución a tantos problemas de convivencia.

Finalmente, el tema de la docencia en educación superior y la valoración de su desempeño permea la totalidad de carreras o programas pertenecientes a una institución de educación superior y, en concordancia con el llamado a contribuir a la comprensión del fenómeno de la docencia en educación superior, emerge concomitantemente una invitación a blandir la investigación y contribuir a la discusión sobre los sistemas de medición de la docencia en educación superior desde la observación y análisis y como su configuración nos permite avanzar en insumos orientados a la mejora de los procesos vinculados a una docencia de calidad.

\section{CONSIDERACIONES FINALES}

Los resultados muestran un total de ocho categorías que evidencian cómo los discursos docentes operan como dispositivos interaccionales entre la práctica pedagógica y los discursos educativos. Estos discursos permiten la construcción de significados que contribuyen a instalar la conceptualización de una cultura 
de la convivencia, es decir, desde las mismas instituciones educativas, se instalan estos discursos internos como dispositivos paradigmáticos relacionados con la práctica educativa, las relaciones educativas y las interacciones pedagógicas que están mediadas desde lógicas que estarían constituyendo en las instituciones escolares una cultura de la Convivencia Escolar (CE).

La CE estaría configurada por elementos formales, como ciertos rituales, definiciones estéticas, rutinas o espacios asignados a determinados objetivos, pero se conforma también a partir de estilos de relación: la presencia o ausencia del afecto en el trato; la manera de abordar las situaciones de sanción; la mayor o menor posibilidad de expresarse que tienen los estudiantes, docentes, apoderados y miembros de la comunidad educativa. Todos estos elementos dan forma y calidad a la convivencia, la afianzan e inciden fuertemente en el nivel de pertenencia de los miembros de la comunidad educativa, que pone en tapete el creer que la convivencia puede lograr procesos de calidad solo si se tiene mejor infraestructura, más y mejores materiales didácticos a disposición de los estudiantes, nuevos criterios de distribución del tiempo escolar, entre otros.

Para nuestros sujetos de investigación, el cumplimiento de la norma y del reglamento por parte de las estudiantes permite calificar positivamente a la convivencia. La convivencia "sana" se asocia a la práctica de una actitud de obediencia a las normativas escolares, esto se evidencia con mayor claridad al referirse a los roles históricos que ellos transfieren a todos los actores que transitan en el espacio escolar y a la valoración de la presencia de los apoderados con el proceso de formación que pretende realizar la escuela. Para ellos, las urgencias agobiantes de su trabajo impiden el desarrollo preventivo de los conflictos que se manifiestan a largo plazo. Así, pues la complejidad de la Convivencia Escolar ha instalado la sensación y percepción de las faltas de herramientas en su proceso de formación como profesionales para tratarla, sumado a las exigencias abrumadoras de la sociedad son factores que determinan e instalan un discurso desesperanzador.

Pareciera que, a primera vista, el mundo escolar se hace amigable para quienes participan en él, sin embargo, se puede desprender, observando más finamente, que existe un quiebre y un espacio que produce incomodidades en la comunidad educativa, que permite establecer diferentes paradigmas en base a componentes implícitos en el discurso de los entrevistados.

Estas construcciones paradigmáticas, implícitas en el discurso de los profesionales de la educación, no son manifestadas discursivamente de manera consciente, se resignifican en sus prácticas cotidianas y le proporcionan una lectura al sentido de sus prácticas, sin que exista una conciencia explicita ni una racionalidad respecto a ello. Sin embargo, se trata de una práctica que da cuenta de que cada sujeto está en un paradigma determinado llámese relacional, normativo o regulativo que se reproduce independientemente del tipo de establecimiento (liceo, colegio o escuela). Estas lógicas escolares se reproducen en los espacios de prácticas educativas de acuerdo con ciertos patrones instalados históricamente. Estos provocan el debilitamiento y el anclaje de modelos de conductas disfuncionales que atentan, llevando al deterioro de la capacidad emocional, la formación de la identidad personal y los compromisos sociales de sus participantes (tanto estudiantes como el resto de la comunidad escolar) y en este sentido, abordar la convivencia escolar pasa, ineludiblemente, por comprender el cómo funciona la escuela, que requiere necesariamente introducirse en el conocimiento de la institución escolar, de sus orígenes y de las diferentes formas de conceptualizarla, que se traducen en modelos.

Finalmente, en relación a "evaluar los discursos que caracterizan y conceptualización de las prácticas educativas de la convivencia escolar", se establece que los profesionales de la educación tienen un discurso instalado sobre aplicar la norma y su regulación como parte fundamental para el manejo del grupo curso, poniendo el acento en los procesos regulatorios que tienen como fin dar forma, formar, instruir y dar un dispositivo castigador para la gestión de la disciplina. En esta misma lógica, las prácticas son parte del desencuentro de la convivencia, es decir, los profesionales educativos generan dispositivos normativos y reguladores, a través de sus prácticas escolares y desde su rol mediador, a partir de los cuales los estudiantes deben seguir un comportamiento que obedezca a los principios y reglas dadas por la cultura institucional. Por ende, la norma denota las prohibiciones y cierra las posibilidades a los estudiantes para que estos expresen 
conductas distintas mencionadas en las normas y reglamentos internos. De esta manera, se legitima la manera "correcta" de comportarse, ser y estar en el mundo escolar, con lo que genera esto denominado cultura de la convivencia escolar.

$\mathrm{Al}$ mismo tiempo, podemos establecer desde los discursos de nuestros entrevistados, la existencia de racionalidades (mediadoras) que intervienen en los procesos de convivencia escolar y que develan una cultura de la convivencia. Esta develación de la cultura de la convivencia no es sólo visibilizar las creencias, sentimientos y pensamientos que se tejen al interior de la escuela en relación a la convivencia, sino comprender las prácticas cotidianas y el ejercicio normativo y regulador en torno a la convivencia que implica lo relacional de la acción educativa en relación a esta, puesto que la cultura de la convivencia es producto de las prácticas cotidianas que se dan en la escuela entre sus diferentes actores, quienes crean y recrean constantemente la convivencia, principal razón que hace que dicha cultura sea un concepto vivo y dialéctico que día a día va siendo modelada por los que son parte de la escuela.

Reconocer la complejidad de sistematizar una cultura de la convivencia es ayudar a su caracterización, ya que se trata de un concepto dinámico y en este sentido, los resultados de esta investigación nos afirman positivamente nuestro supuesto de que existe una "cultura de la Convivencia Escolar", definido como un espacio interaccional en donde se intercambian experiencias, se construyen y reconstruyen significados a través de las prácticas. En este sentido, se puede afirmar que existe una cultura de la convivencia escolar como institución permanente, que se produce y reproduce a partir de las dinámicas endógenas y exógenas de las instituciones educativas en el marco de racionalidades internas que estarían compuestas de relaciones, normas y regulaciones, que permean todo el espacio escolar y que no están escritas en los propios manuales de convivencia. Esta cultura captura a toda la organización en su conjunto acerca de cómo entender la convivencia, es decir, que la convivencia estaría siendo resignificada a partir de las ideas expresadas en los discursos simbólicos que se transmiten a cada sujeto adulto que habita la escuela y que constituyen la manera de concebir la convivencia desde lo relacional, lo normativo y lo regulativo. Este sistema de significados forma parte de lo que la gente piensa y la forma en que actúa para vivir la convivencia. Asimismo, tiene características estáticas y dinámicas que se ponen de manifiesto, por un lado, para crear un carácter único del sistema escolar al promover un sentido relacional, normativo y regulativo; por otro, para introducir a los nuevos miembros en una particular racionalización de cómo vivir la convivencia. En este encuadre, la cultura de la convivencia se define como un sistema de significados que es fruto de las interacciones sociales entre los miembros de manera uniforme, que representa la identidad de la organización escolar.

Esta investigación permitió sistematizar la caracterización de la cultura de la convivencia como modelo socialmente organizado e identificar las barreras entre el ámbito profesional y el personal, pues las decisiones de las personas no se sustentan en su conocimiento explícito profesional, sino en sus modos de pensar, sentir y actuar instaladas en las mismas instituciones escolares, que a su vez, instalan en cada profesional educativo una construcción paradigmática sobre cómo entender la convivencia escolar.

\section{REFERENCIAS}

Acosta, A. (2003). Resolución de conflictos y regulación de sentimientos. Actas del I Congreso Hispanoamericano de Educación y Cultura de Paz, 293-304. Granada.

Carbajal Padilla, P. (2013). Convivencia democrática en las escuelas. Apuntes para una reconceptualización. Revista Iberoamericana de Evaluación Educativa, 6(2). Recuperado de https://revistas.uam.es/riee/article/view/3403

Cerda, G.; Salazar, Y.; Guzmán, C. \& Narváez, G. (2018). Impacto de la convivencia escolar sobre el rendimiento académico, desde la percepción de estudiantes con desarrollo típico y necesidades educativas especiales. Propósitos $y$ Representaciones, 6(1), 247-300. http://dx.doi.org/10.20511/pyr2018.v6n1.194

Fierro-Evans, C. \& Carbajal-Padilla, P. (2019). Convivencia Escolar: Una revisión del concepto. Psicoperspectivas, 18(1), 9-27. https://dx.doi.org/10.5027/psicoperspectivas-vol18-issue1-fulltext-1486 
Fuquen Alvarado, M.E. (2003). Los conflictos y las formas alternativas de resolución. Tabula Rasa, (1), 265-278. Recuperado de http://www.redalyc.org/articulo.oa?id=39600114

Güell i Barceló, M. y Muñoz Redon J. (2000). Desconócete a ti mismo. Programa de alfabetización emocional. Barcelona: Paidós

Gutiérrez-Méndez, D., \& Pérez-Archundia, E. (2015). Estrategias para generar la convivencia escolar. Ra Ximhai, 11(1), 63-81. Recuperado de http://www.redalyc.org/comocitar.oa?id=46139401004

Hernández, R.; Fernández, C. y Baptista, M.P. (2006). Metodología de la investigación (quinta edición). México: McGraw-Hill

Jimerson, S. \& Furlong, M. (2006). The handbook of school violence and school safety. From research to practice. Mahwah, New Jersey. Lawrence Erlbaum Associates

Laboratorio Latinoamericano de Evaluación de la Calidad de la Educación (LLECE) (2002) Qualitative Study of Schools with Outstanding Results in Seven Latin American Countries. Santiago: OREALC/UNESCO Santiago.

Maldonado, J. (2004). Convivencia escolar: ensayos y experiencias. Buenos Aires: Lugar Editorial.

Marías, J. (1996). Persona. Madrid: Alianza editorial.

Ministerio de Educación de Chile (2005). Metodologías de trabajo para el mejoramiento de la calidad de la convivencia escolar.

Ministerio de Educación de Chile (2010). Conviviendo mejor en la escuela y en el liceo. Manual sobre convivencia escolar dirigido a todos los integrantes de la comunidad educativa.

Ministerio de Educación de Chile (2015). Política Nacional de Convivencia Escolar. Santiago: MINEDUC

Muñoz, MT.; Lucero, B.; Cornejo, C.; Muñoz, P. \& Araya, N. (2014). Convivencia y clima escolar en una comunidad educativa inclusiva de la Provincia de Talca, Chile. Revista electrónica de investigación educativa, 16(2), 16-32.

Nail Kröyer, O.; Muñoz Reyes, M. \& Gajardo Aguayo, J. (2013). Principios orientadores de la convivencia en el aula: una estrategia de reflexión colectiva. Educação e Pesquisa, 39(2), 367-385. https://dx.doi.org/10.1590/ S1517-97022013000200006

Ochoa, A. \& Diez-Martín, E. (2013). El reglamento escolar como eje de Análisis de la convivencia en la escuela. Ensaio: Avaliação e Políticas Públicas em Educação, 21(81), 667-684, http://dx.doi.org/10.1590/ S0104-40362013000400003

Ortega, R. (2007). Competencias para la convivencia y las relaciones sociales. Cuadernos de pedagogía, 370, 32-35.

Ramírez, S. \& Justicia, F. (2006). El maltrato entre escolares y otras conductas-problema para la convivencia. Revista Electrónica de Investigación Psicoeducativa, 9(4), 265-290.

Sandoval, M. (2014). Convivencia y clima escolar: claves de la gestión del conocimiento. Última década, 22(41), 153-178. https://dx.doi.org/10.4067/S0718-22362014000200007

Serey, D. (2006). Las nuevas formas de la discriminación y la estigmatización. El caso del concepto bullying. Revista Repsi, 87, 8-14.

Serey, D. (2011). Bullying. El fenómeno del matonaje escolar: Teoría y aplicaciones preventivas en la escuela. Santiago de Chile: Editorial Mataquito.

Serey, D. (2013). Cómo abordar la convivencia en las instituciones escolares: Un modelo Didáctico para organizar la Convivencia Escolar. Saarbrücken, Germany: Editorial Académica Española.

Serey, D. (2019). Cultura de la Convivencia Escolar: Estudio de las racionalidades (Mediadora) sobre convivencia escolar en los profesionales de la educación municipal de la Comuna de Puente Alto (Tesis doctoral). Universidad de la Integración de las Américas, Paraguay.

Tuvilla, J. (2004). Convivencia escolar y resolución pacifica de conflictos. Sevilla: Consejería de educación y ciencia primaria, Junta de Andalucía.

Valenzuela, J.; Ahumada, I.; Rubilar, A.; López, V. \& Urbina, C. (2018). El encargado de convivencia escolar en Chile: hacia la comprensión de su identidad laboral. Revista de Psicología, 36(1), 189-216. https:// dx.doi.org/10.18800/psico.201801.007 
Daniel Serey Araneda. Percepciones de los profesionales educativos sobre la Convivencia Escolar

Van den Berg, G. (2001). Duration models: specification, identification and multiple durations. In Heckman, J.J. \& Leamer, E.E. (ed.). Handbook of Econometrics, volume 5 (3381-3460). North Holland: Elsevier.

Vilar Rubiano, D.M. y Carretero, A. (2008). Vivir convivir: convivencia intercultural en centros de educación. Barcelona: Paidós.

\section{BY-ND}

